University of Wollongong

Research Online

Faculty of Engineering and Information

Faculty of Engineering and Information

Sciences - Papers: Part A

Sciences

$1-1-2013$

Difference between far-infrared photoconductivity spectroscopy and absorption spectroscopy: theoretical evidence of the electron reservoir mechanism

\author{
Tadashi Toyoda \\ Tokai University \\ Maho Fujita \\ Tokai University \\ Tomohisa Uchida \\ Tokai University \\ Nobuyoshi Hiraiwa \\ Fujitsu Component Limited \\ Taturo Fukuda \\ Japan Electronics College
}

See next page for additional authors

Follow this and additional works at: https://ro.uow.edu.au/eispapers

Part of the Engineering Commons, and the Science and Technology Studies Commons

Research Online is the open access institutional repository for the University of Wollongong. For further information contact the UOW Library: research-pubs@uow.edu.au 


\title{
Difference between far-infrared photoconductivity spectroscopy and absorption spectroscopy: theoretical evidence of the electron reservoir mechanism
}

\author{
Abstract \\ The intriguing difference between far-infrared photoconductivity spectroscopy and absorption \\ spectroscopy in the measurement of the magnetoplasmon frequency in GaAs quantum wells reported by \\ Holland et al. [Phys. Rev. Lett. 93, 186804 (2004)] remains unexplained to date. This Letter provides a \\ consistent mechanism to solve this puzzle. The mechanism is based on the electron reservoir model for \\ the integer quantum Hall effect in graphene [Phys. Lett. A 376, 616 (2012)]. We predict sharp kinks to \\ appear in the magnetic induction dependence of the magnetoplasmon frequency at very low \\ temperatures such as $14 \mathrm{mK}$ in the same GaAs quantum well sample used by Holland et al..

\section{Keywords} \\ far, infrared, photoconductivity, spectroscopy, absorption, theoretical, evidence, difference, electron, \\ between, reservoir, mechanism

\section{Disciplines} \\ Engineering | Science and Technology Studies \\ Publication Details \\ Toyoda, T., Fujita, M., Uchida, T., Hiraiwa, N., Fukuda, T., Koizumi, H. \& Zhang, C. (2013). Difference \\ Between Far-Infrared Photoconductivity Spectroscopy and Absorption Spectroscopy: Theoretical \\ Evidence of the Electron Reservoir Mechanism. Physical Review Letters, 111 (8), 086801-1-086801-4.

\section{Authors} \\ Tadashi Toyoda, Maho Fujita, Tomohisa Uchida, Nobuyoshi Hiraiwa, Taturo Fukuda, Hideki Koizumi, and \\ Chao Zhang
}




\title{
Difference Between Far-Infrared Photoconductivity Spectroscopy and Absorption Spectroscopy: Theoretical Evidence of the Electron Reservoir Mechanism
}

\author{
Tadashi Toyoda, ${ }^{1, *}$ Maho Fujita, ${ }^{1}$ Tomohisa Uchida, ${ }^{1}$ Nobuyoshi Hiraiwa, ${ }^{2}$ Taturo Fukuda, ${ }^{3}$ \\ Hideki Koizumi, ${ }^{4}$ and Chao Zhang ${ }^{5}$ \\ ${ }^{1}$ Department of Physics, Tokai University, Kitakaname 4-1-1, Hiratsuka, Kanagawa 259-1292, Japan \\ ${ }^{2}$ Fujitsu Component Limited, Oazasuzaka 1174, Suzaka, Nagano 382-0076, Japan \\ ${ }^{3}$ Japan Electronics College, Hyakunin-cho 1-25-4, Shinjuku, Tokyo 169-8522, Japan \\ ${ }^{4}$ Nippon Gear Co., Ltd., Kirihara-cho 7, Fujisawa, Kanagawa 252-0811, Japan \\ ${ }^{5}$ Institute of Superconducting and Electronic Materials and School of Physics, University of Wollongong, \\ New South Wales 2522, Australia \\ (Received 24 April 2013; published 20 August 2013)
}

\begin{abstract}
The intriguing difference between far-infrared photoconductivity spectroscopy and absorption spectroscopy in the measurement of the magnetoplasmon frequency in GaAs quantum wells reported by Holland et al. [Phys. Rev. Lett. 93, 186804 (2004)] remains unexplained to date. This Letter provides a consistent mechanism to solve this puzzle. The mechanism is based on the electron reservoir model for the integer quantum Hall effect in graphene [Phys. Lett. A 376, 616 (2012)]. We predict sharp kinks to appear in the magnetic induction dependence of the magnetoplasmon frequency at very low temperatures such as $14 \mathrm{mK}$ in the same GaAs quantum well sample used by Holland et al..
\end{abstract}

DOI: 10.1103/PhysRevLett.111.086801

PACS numbers: 73.20.Mf, 73.21.- b, 73.50.-h

Although most of the transport and optical measurements in low-dimensional semiconductors can be successfully explained by various theoretical models, there remain a few experiments where a full understanding is still missing. The pioneering work of Holland et al. [1] is one of those experiments where a consistent theory is lacking. The key finding of that experiment, which is yet to be understood, is the qualitative difference between magnetoplasmon frequency measured in far-infrared photoconductivity (FIR-PC) spectroscopy and that measured in absorption spectroscopy. This difference was unexpected then and remains unexplained to this date. The purpose of this Letter is to solve this puzzle using a theory based on the electron reservoir model (ERM).

Among many theoretical models of the integer quantum Hall effect [2], the ERM [3-7] can reproduce the quantum Hall resistivity as a function of the gate voltage, magnetic induction, and temperature in perfect agreement with experiment. Nevertheless, the original idea [3] that the electron reservoir (ER) is due to donor impurity levels had been believed unlikely [8]. The unknown mechanism of the ER had been obscuring the validity of the ERM for decades until it was finally revealed very recently [6]. It was found that the mechanism can be explained by considering nonuniform electron density distribution in the two-dimensional electron system (2DES) caused by the Lorentz force. This can happen only when there are both a dc current and a perpendicular magnetic field in the 2DES [6]. The proposed mechanism should provide an important missing piece in the ERM for the integer quantum Hall effect. It is, therefore, of paramount significance to verify the correctness of the proposed mechanism by carefully examining as many different experiments as possible. In this Letter we investigate the measurement of magnetoplasmon frequency by two different experimental methods carried out by Holland et al. [1] and show that their findings provide clear theoretical evidence for the mechanism of the ER proposed in [6]. We also propose a new experiment that would validate the proposed mechanism of the electron reservoir model.

Holland et al. [1] measured the long-wavelength magnetoplasmon frequency in a GaAs quantum well by varying the magnetic induction. Using both FIR-PC spectroscopy and absorption spectroscopy, they explored a wide range of magnetic induction strengths to obtain explicit filling factor dependence of the magnetoplasmon dispersion. They found that the dispersion measured in the FIR-PC spectroscopy deviates quite significantly from the well-established semiclassical dispersion. They defined the renormalized magnetoplasmon frequency,

$$
\Omega_{m p}^{\mathrm{EXP}}=\frac{\left\{\omega_{m p}^{\mathrm{EXP}}\right\}^{2}-\omega_{c}^{2}}{\omega_{c}}=\frac{2 \pi e c N_{s}}{\varepsilon B} q=\frac{2 \pi e^{2}}{\varepsilon h} \nu q,
$$

where $\omega_{m p}^{\mathrm{EXP}}$ is the measured magnetoplasmon frequency, $\varepsilon$ is the dielectric constant of the GaAs semiconductor into which the 2DES is embedded, $-e$ is the electron charge, $N_{s}$ is the electron number density, $q$ is the wave number vector, $\omega_{c}=e B / m c$ is the cyclotron frequency, $m$ is the electron effective mass, and $\nu=h c N_{s} / e B$ is the filling factor. For a fixed value of the wave number vector $q$, Eq. (1) would show that $\Omega_{m p}^{\mathrm{EXP}}$ is simply proportional to the filling factor. After the measurement, however, they found a quantized dispersion with plateaus forming around even filling factors in the FIR-PC measurement. Such plateaus are not observed 
in the absorption spectroscopy measurement. The mechanism of the observed magnetoplasmon plateaus has been explained by the electron reservoir model [9], but the origin of the difference between the two methods, i.e., FIR-PC and absorption spectroscopy, has been left unsolved. In this Letter we theoretically verify that the difference is due to the presence of dc current in the FIR-PC measurement. We also calculate the magnetic induction dependence of the magnetoplasmon dispersion at $14 \mathrm{mK}$. Our calculation predicts sharp kinks in the magnetoplasmon frequency as a function of the magnetic induction for a fixed value of the wavelength if FIR-PC spectroscopy is used. Such kinks should not be observed in the absorption spectroscopy measurement.

Quantum statistical calculation of the dispersion of the magnetoplasmon has been carried out by many authors. One of the simplest theories is the self-consistent linear response approximation (SCLRA) [10]. In classical electrodynamics the scalar potential $A_{0}^{(1)}$ induced by the electron density fluctuation $\delta \rho$ satisfies the Poisson equation

$$
\nabla^{2} A_{0}^{(1)}(\boldsymbol{x}, t)=4 \pi e \epsilon^{-1} \delta \rho(\boldsymbol{x}, t) .
$$

In the SCLRA the density fluctuation on the right-hand side is assumed to be given by the linear response formula

$$
\begin{aligned}
& \delta \rho(\boldsymbol{x}, t) \\
& \quad=\frac{-e}{\hbar} \int d t^{\prime} \int d^{3} \boldsymbol{x}^{\prime}\left|\chi\left(x_{3}\right) \chi\left(x_{3}^{\prime}\right)\right|^{2} \mathcal{D}\left(\boldsymbol{r}^{\prime}, t ; \boldsymbol{r}^{\prime}, t^{\prime}\right) A_{0}^{(1)}\left(\boldsymbol{x}^{\prime}, t^{\prime}\right),
\end{aligned}
$$

where $\mathcal{D}\left(\boldsymbol{r}, t ; \boldsymbol{r}^{\prime}, t^{\prime}\right)$ is the retarded density response function of two-dimensional electrons and $\chi\left(x_{3}\right)$ is the localized wave function along the direction perpendicular to the quantum well. We use the notation $\boldsymbol{r} \equiv\left(x_{1}, x_{2}\right)$. An integro-differential equation for the density fluctuation with retardation effects can be derived by eliminating the scalar potential $A_{0}^{(1)}$ from Eqs. (2) and (3). By expanding the Fourier transform of the electron density response function in powers of the wave vector $q$, the equation can be analytically solved and the dispersion of the magnetoplasmon can be calculated [10-14]. Considering up to the fourth order terms, we obtain two magnetoplasmon modes [14]:

$$
\frac{\omega_{I}^{2}}{\omega_{c}^{2}}=1+\kappa\left\{A_{1} l q-\frac{A_{2}}{2}(l q)^{3}-\frac{A_{1} A_{2}}{6} \kappa(l q)^{4}\right\}
$$

and

$$
\frac{\omega_{I I}^{2}}{\omega_{c}^{2}}=4+\frac{\kappa A_{2}}{2}(l q)^{3}+\frac{A_{1} A_{2}}{6} \kappa^{2}(l q)^{4}
$$

where the wave number vector $q$, the magnetic length $l=\sqrt{c \hbar / e B}$, and $\kappa=e^{2} / \epsilon l \hbar \omega_{c}$ have been defined. The coefficients $A_{1}$ and $A_{2}$ are given by the Fermi distribution function as

$$
A_{1}=\sum_{\alpha} \sum_{n=0}^{\infty} \frac{1}{1+\exp \beta\left(E_{n \alpha}-\mu\right)}
$$

and

$$
A_{2}=\sum_{\alpha} \sum_{n=0}^{\infty} \frac{2 n+1}{1+\exp \beta\left(E_{n \alpha}-\mu\right)},
$$

where $\beta=1 / k_{B} T$ and $E_{n \alpha}$ is the energy eigenvalue of the $n$th Landau level with spin $\alpha$, given by

$$
E_{n \alpha}=\hbar \omega_{c}\left(n+\frac{1}{2}\right)+\frac{g^{*}}{2} \mu_{B} B \operatorname{sgn}(\alpha) .
$$

Here we have defined the Bohr magneton $\mu_{B}=e \hbar / 2 m_{0} c$ with the electron rest mass $m_{0}$, and the effective $g$ factor $g^{*}$. We adopt $g^{*}=6$ in this work [9]. We have also defined $\operatorname{sgn}(\alpha)=\alpha /|\alpha|$ for the spin variable $\alpha$. In the above calculation we assumed the zero-thickness limit for the wave function $\chi$. The effects of finite thickness are discussed in Ref. [14].

In the geometry of the magnetoplasmon experiment shown in Fig. 1, the magnetic field is perpendicular to the $x_{1}-x_{2}$ plane on which the 2DES is placed. In the FIRPC measurement there is a steady dc current $I_{1}$ in the $x_{1}$ direction, because the measurement of resistivity requires dc current. By virtue of the Lorentz force acting on the electrons there is a macroscopic accumulation of electrons on one side if there is a macroscopic steady flow of the electrons. Consequently, in order to analyze the FIR-PC data it is necessary to take into account the nonuniform spatial distribution of the electrons along the $x_{2}$ direction. On the other hand, there is no such current in the absorption spectroscopy measurement. This is the essential difference between the two experimental methods.

The nonuniform charge density can be taken into account by dividing the 2DES into many strips of rectangular-shaped subsystems parallel to the $x_{1}$ axis as shown in Fig. 1 [6]. The length and width of each subsystem are $L_{1}$ and $\Delta L$, respectively. A subsystem is denoted by $S^{i}\left(i=1,2,3, \ldots, L_{2} / \Delta L\right)$. We assume the macroscopic condition $a \ll \Delta L \ll L_{2}$, where $a$ is the

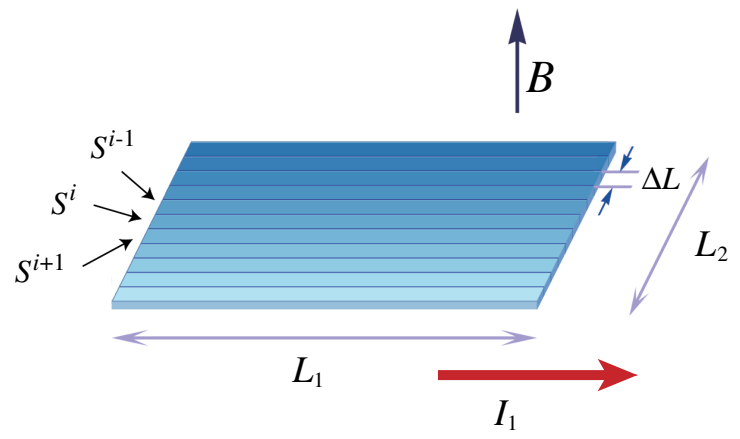

FIG. 1 (color online). The geometry of the 2DES under perpendicular uniform static magnetic induction field $\boldsymbol{B}$ and a steady dc current $\boldsymbol{I}=\left(I_{1}, 0,0\right)$. The rectangular-shaped subsystems parallel to the $x_{1}$ axis are denoted by $S^{i}$. 


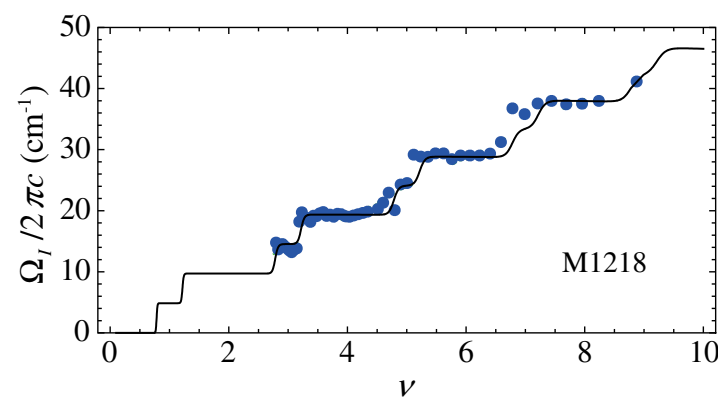

FIG. 2 (color online). The solid line is the filling factor dependence of the renormalized magnetoplasmon dispersion calculated by Eq. (10) using the parameters for the sample M1218 given in [1], i.e., $N_{s}=5.58 \times 10^{11} \mathrm{~cm}^{-2}$ and $\epsilon=15$. The dots are experimental data taken from Fig. 4(a) of Ref. [1].

mean free path of the electrons and $L_{2}$ is the width of the 2DES. The spatial distribution of the electron number density in each subsystem is assumed to be uniform. Their values may depend on $i$, but the chemical potential should take the same value. This is the mechanism of the electron reservoir model proposed by Toyoda and Zhang [6] to explain the integer quantum Hall effect observed in graphene. The SCLRA can be applied to each subsystem to calculate the magnetoplasmon dispersion. Because the total photon energy of the electromagnetic radiation due to the magnetoplasmons from all subsystems is measured in the experiment [1], the observed magnetoplasmon dispersion is given by the dispersion of each subsystem. Hence the dispersion is given by Eqs. (4) and (5). The theoretical difference between the observed dispersions in the FIRPC spectroscopy and absorption spectroscopy measurements can be attributed to the quantum statistical expectation value for the electron number density:

$$
\frac{e B}{h c} \sum_{\alpha} \sum_{n=0}^{\infty} \frac{1}{1+\exp \beta\left(E_{n \alpha}-\mu\right)}=N_{2 \mathrm{DES}} .
$$

In the FIR-PC measurement the chemical potential is a given quantity and $N_{2 \mathrm{DES}}$ becomes a function of $B, T$, and $\mu$. On the other hand, in the absorption spectroscopy measurement $N_{2 \mathrm{DES}}$ is a given quantity. Consequently, the $B$ dependence of the coefficients $A_{1}$ and $A_{2}$ defined by Eqs. (6) and (7) is totally different in the two measurements. For the FIR-PC measurement the chemical potential is given as $\mu=\pi \hbar^{2} N_{s} / m$, where $N_{s}$ is the electron number density of the sample [7,9]. The dispersions (4) and (5) are independent of the size of each subsystem. Each subsystem may have different electron number density, but the chemical potential remains constant over the whole system. Therefore, if the SCLRA is applied to each subsystem, the total dispersion is equivalent to Eqs. (4) and (5).

In Figs. 2 and 3 we plot the renormalized dispersion

$$
\frac{\Omega_{I}}{2 \pi c}=\frac{\omega_{I}^{2}-\omega_{c}^{2}}{2 \pi c \omega_{c}}=\frac{\kappa \omega_{c} l q}{2 \pi c}\left[A_{1}-\frac{A_{2}}{2}(l q)^{2}-\frac{A_{1} A_{2} \kappa}{6}(l q)^{3}\right]
$$

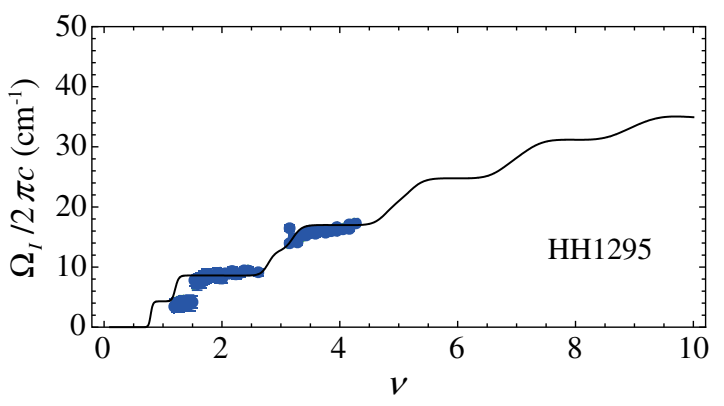

FIG. 3 (color online). The solid line is the filling factor dependence of the renormalized magnetoplasmon dispersion calculated by Eq. (10) using the parameters for the sample HH1295 given in [1], i.e., $N_{s}=1.93 \times 10^{11} \mathrm{~cm}^{-2}$ and $\epsilon=16.9$. The dots are experimental data taken from Fig. 4(b) of Ref. [1].

as a function of the filling factor $\nu=h c N_{s} / e B$ for the sample M1218 and HH1295 in the measurement by Holland et al. [1]. The electron number densities are $N_{s}=$ $5.58 \times 10^{11} \mathrm{~cm}^{-2}$ for M1218 and $N_{s}=1.93 \times 10^{11} \mathrm{~cm}^{-2}$ for HH1295 [1]. The dielectric constant $\epsilon$ in $\kappa$ is calculated using the value of the Thomas-Fermi wave vector given in [1], i.e., $\epsilon=15$ for M1218 and $\epsilon=16.9$ for HH1295. In both Figs. 2 and 3 the experimental data taken from Fig. 4 in Ref. [1] are given by blue dots which show excellent agreement with the theoretical results. This calculation improves our previous results given in Ref. [9] as the renormalized dispersion (10) includes the higher order terms in the power series expansion of the wave vector. As shown in [14], the long wavelength expansion is valid for $B_{c}<B$ with $B_{c} \simeq 2$ (T) for the experimental condition of Ref. [1]. This may explain the deviation of the theoretical renormalized

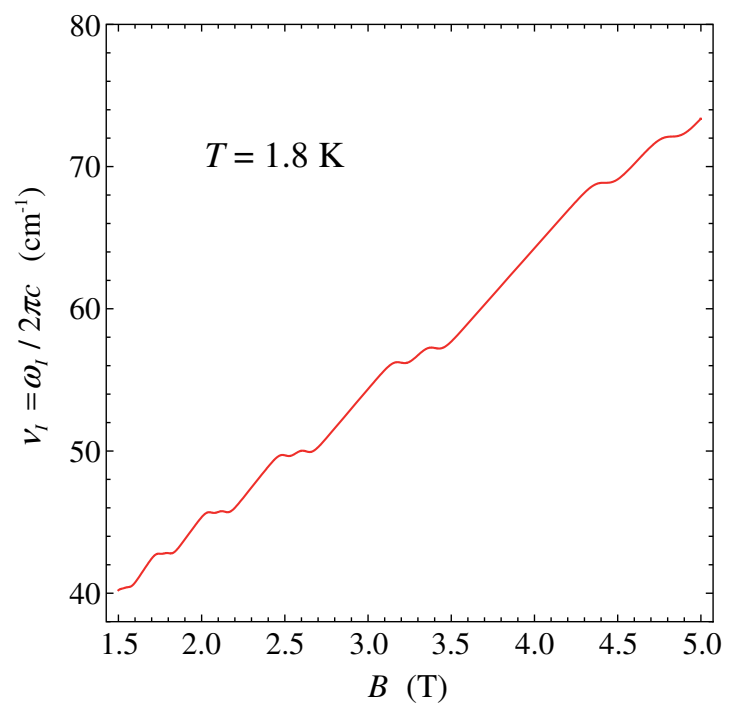

FIG. 4 (color online). The $B$-field dependence of the magentoplasmon dispersion $\nu_{I}=\omega_{I} / 2 \pi c$ calculated by Eq. (4) for $T=1.8 \mathrm{~K}$. The parameters for the sample M1218 given in [1], i.e., $N_{s}=5.58 \times 10^{11} \mathrm{~cm}^{-2}$ and $\epsilon=15$, are used. 


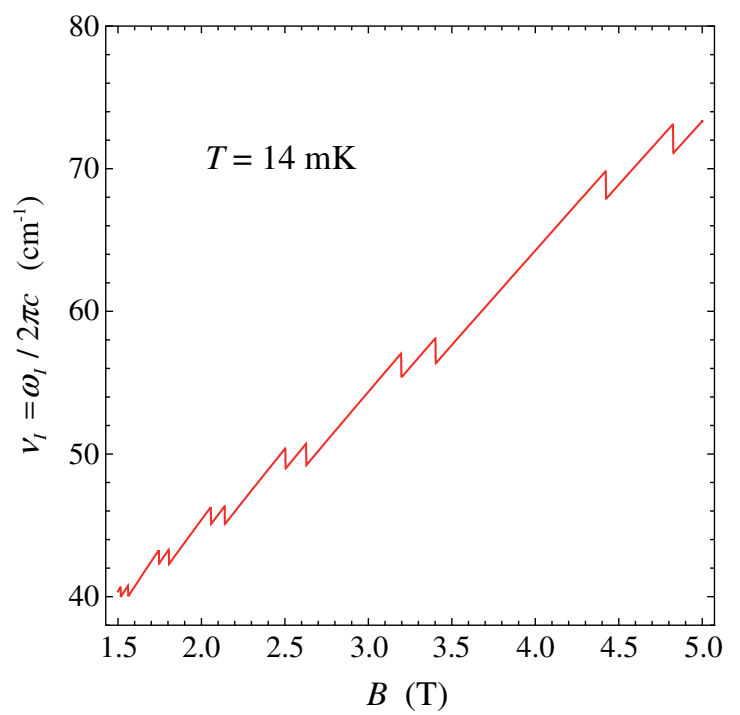

FIG. 5 (color online). The $B$-field dependence of the magentoplasmon dispersion $\nu_{I}=\omega_{I} / 2 \pi c$ calculated by Eq. (4) for $T=14 \mathrm{mK}$. The parameters for the sample M1218 given in [1], i.e., $N_{s}=5.58 \times 10^{11} \mathrm{~cm}^{-2}$ and $\epsilon=15$, are used.

dispersion for the small values of the magnetic induction in Fig. 3.

In Fig. 4 we plot the magnetic induction dependence of the magnetoplasmon frequency given by (4), using the values of the parameters of the sample M1218 given in Ref. [1] at $T=1.8 \mathrm{~K}$. The theoretical dispersion curve clearly shows the characteristic deviations as found in the experimental data given in Fig. 3 of Ref. [1]. The agreement with the experiment is excellent. It should be noted that Figs. 2 and 3 are for the renormalized magnetoplasmon frequencies given by (10), while Figs. 4-6 are for the magnetoplasmon frequencies given by (4). The plateaus in the renormalized frequency plot become the peak structure in the frequency plot.

In Fig. 5 we plot the same dispersion for $T=14 \mathrm{mK}$. As shown in Fig. 5 there appear distinct sharp kinks in the $B$ dependence of the dispersion at $T=14 \mathrm{mK}$. By comparing the magnetoplasmon frequency measured at $14 \mathrm{mK}$ with that measured at $1.8 \mathrm{~K}$, one should be able to observe these kinks predicted here. Therefore, we propose an extremely low temperature measurement of the magnetic induction dispersion by using the FIR-PC spectroscopy as well as the absorption spectroscopy for a GaAs quantum well sample such as M1250 or HH1295 used in the experiment by Holland et al. [1]. Our theory predicts that only the FIR-PC spectroscopy measurement will produce the sharp kinks in the dispersion.

In conclusion, we have shown that a collective excitation energy such as magnetoplasmon frequency can be qualitatively different if it is measured by FIR-PC spectroscopy or deduced from absorption spectroscopy. This difference can be attributed to the fact that a dc current exists in the

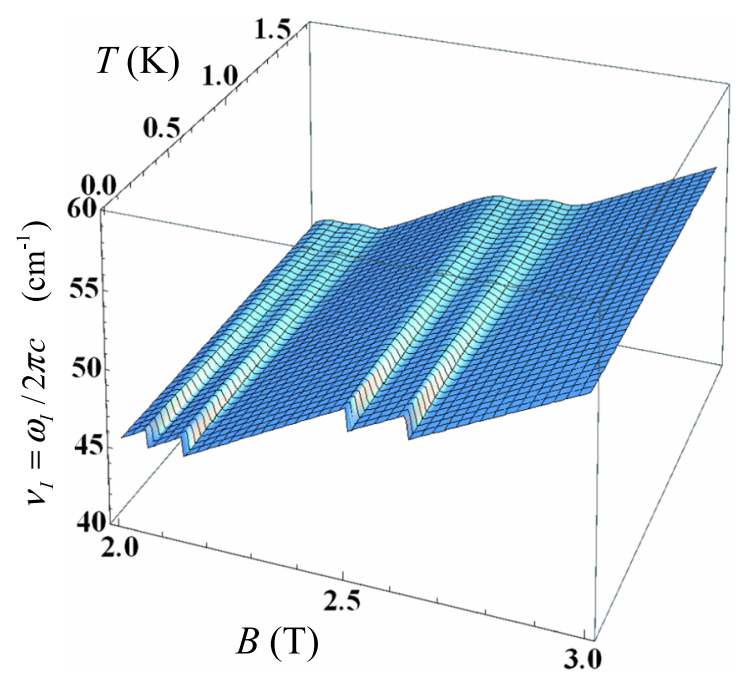

FIG. 6 (color online). The magentoplasmon dispersion $\nu_{I}=$ $\omega_{I} / 2 \pi c$ given by Eq. (4) is plotted as a function of the magnetic induction $B$ and temperature $T$. The parameters for the sample M1218 given in [1], i.e., $N_{s}=5.58 \times 10^{11} \mathrm{~cm}^{-2}$ and $\epsilon=15$, are used.

FIR-PC experiment and is absent in the absorption spectroscopy. The Lorentz force acting on the dc current causes nonuniform electron density distribution, and therefore the chemical potential should be adopted as the relevant thermodynamic variable to characterize the 2DES under a perpendicular magnetic field. This mechanism can only be consistently analyzed with the ER model [6].

We thank Professor Masaki Yasue for helpful comments.

*toyoda@keyaki.cc.u-tokai.ac.jp

[1] S. Holland, Ch. Heyn, D. Heitmann, E. Batke, R. Hey, K. J. Friedland, and C.-M. Hu, Phys. Rev. Lett. 93, 186804 (2004).

[2] K. von Klitzing, G. Dorda, and M. Pepper, Phys. Rev. Lett. 45, 494 (1980).

[3] G. A. Baraff and D. C. Tsui, Phys. Rev. B 24, 2274 (1981).

[4] T. Toyoda, V. Gudmundsson, and Y. Takahashi, Phys. Lett. 102A, 130 (1984).

[5] T. Toyoda, V. Gudmundsson, and Y. Takahashi, Physica (Amsterdam) 132A, 164 (1985).

[6] T. Toyoda and C. Zhang,Phys. Lett. A 376, 616 (2012).

[7] K. Yamada, T. Uchida, J. Iizuka, M. Fujita, and T. Toyoda, Solid State Commun. 155, 79 (2013).

[8] K. von Klitzing, Rev. Mod. Phys. 58, 519 (1986).

[9] T. Toyoda, N. Hiraiwa, T. Fukuda, and H. Koizumi, Phys. Rev. Lett. 100, 036802 (2008).

[10] T. Toyoda, Physica (Amsterdam) 253A, 498 (1998).

[11] T. Fukuda, N. Hiraiwa, T. Mitani, and T. Toyoda, Phys. Rev. B 76, 033416 (2007).

[12] T. Fukuda and T. Toyoda, Phys. Rev. B 70, 205117 (2004).

[13] T. Toyoda and T. Fukuda, Phys. Rev. B 71, 205312 (2005).

[14] T. Uchida, N. Hiraiwa, K. Yamada, J. Iizuka, M. Fujita, and T. Toyoda, arXiv:1307.5578. 